\title{
On solvability of the cohomology equation in function spaces
}

\author{
by \\ Ryotaro Sato (Okayama)
}

\begin{abstract}
Let $T$ be an endomorphism of a probability measure space $(\Omega, \mathcal{A}, \mu)$, and $f$ be a real-valued measurable function on $\Omega$. We consider the cohomology equation $f=h \circ T-h$. Conditions for the existence of real-valued measurable solutions $h$ in some function spaces are deduced. The results obtained generalize and improve a recent result of Alonso, Hong and Obaya.
\end{abstract}

1. Introduction. Let $(\Omega, \mathcal{A}, \mu)$ be a probability measure space and $T: \Omega \rightarrow \Omega$ be an endomorphism of $(\Omega, \mathcal{A}, \mu)$. Thus, if $A \in \mathcal{A}$ then $T^{-1} A \in \mathcal{A}$ and $\mu\left(T^{-1} A\right)=\mu(A)$. $T$ is called an automorphism of $(\Omega, \mathcal{A}, \mu)$ if $T$ is oneto-one and onto, and $T^{-1}$ is again an endomorphism of $(\Omega, \mathcal{A}, \mu)$. If there does not exist a set $A$ in $\mathcal{A}$ with $T^{-1} A=A$ and $0<\mu(A)<1$, then $T$ is called ergodic. Let $f$ be a real-valued measurable function on $\Omega$. Then we define

$$
S_{0} f(\omega)=0 \quad \text { and } \quad S_{j} f(\omega)=\sum_{k=0}^{j-1} f\left(T^{k} \omega\right) \quad \text { for } j \geq 1,
$$

so that the cocycle identity $S_{j+k} f(\omega)=S_{j} f(\omega)+S_{k} f\left(T^{j} \omega\right)$ holds for each $j, k \geq 0$. The function $f$ is called a coboundary cocycle if there exists a real-valued measurable function $h$ on $\Omega$ such that

$$
f(\omega)=h(T \omega)-h(\omega) \quad \text { for } \mu \text {-a.e. } \omega \in \Omega .
$$

In this case we have

$$
S_{j} f(\omega)=h\left(T^{j} \omega\right)-h(\omega) \quad \text { for } \mu \text {-a.e. } \omega \in \Omega
$$

for all $j \geq 1$.

Recently Alonso, Hong and Obaya [1] considered the case where $T$ is an ergodic automorphism and $f$ is a function in $L_{r}(\Omega, \mu)$ with $0<r<\infty$.

2000 Mathematics Subject Classification: 37A20, 28D05, 47A35.

Key words and phrases: probability measure space, endomorphism, automorphism, ergodic, cohomology equation, real coboundary cocycle, Banach lattice of equivalence classes of real-valued measurable functions. 
They proved that if

$$
\liminf _{n \rightarrow \infty} \frac{1}{n} \sum_{j=1}^{n} \int_{A}\left|S_{j} f\right|^{r} d \mu<\infty
$$

for some $A \in \mathcal{A}$ with $\mu(A)>0$, then there exists a function $h$ in $L_{r}(\Omega, \mu)$ such that

$$
f(\omega)=h(T \omega)-h(\omega) \quad \text { for } \mu \text {-a.e. } \omega \in \Omega .
$$

For related results we refer the reader to [2] and [11]. In the present paper we intend to generalize and improve the result of [1]. We prove in Section 3 that if $T$ is an ergodic endomorphism, and $\varphi: \mathbb{R} \rightarrow[0, \infty)$ is a Borel measurable function on the real line $\mathbb{R}$ such that $\lim _{|x| \rightarrow \infty} \varphi(x)=\infty$, then the condition

$$
\liminf _{n \rightarrow \infty} \frac{1}{n} \sum_{j=1}^{n} \int_{A} \varphi\left(S_{j} f(\omega)\right) d \mu<\infty
$$

for some $A \in \mathcal{A}$ with $\mu(A)>0$ implies the existence of a real-valued measurable function $h$ on $\Omega$ such that $f(\omega)=h(T \omega)-h(\omega)$ for $\mu$-a.e. $\omega \in \Omega$. If the function $\varphi$ satisfies the additional hypotheses that $\sup \{\varphi(x):|x| \leq N\}<\infty$ for every $N \geq 1$ and $\lim \sup _{|x| \rightarrow \infty} \varphi(x+a) / \varphi(x)<\infty$ for every $a \in \mathbb{R}$, then $\int_{\Omega} \varphi(h(\omega)) d \mu<\infty$. Secondly we consider a Banach lattice $\left(L,\|\cdot\|_{L}\right)$ of equivalence classes of real-valued measurable functions on $\Omega$. Under suitable conditions on $L$, we prove that if $T$ is an ergodic endomorphism, and there exists a set $A$ in $\mathcal{A}$ with $\mu(A)>0$ such that $\left(S_{j} f\right) \chi_{A} \in L$ for $j \geq 1$ and

$$
\liminf _{n \rightarrow \infty} \frac{1}{n} \sum_{j=1}^{n}\left\|\left(S_{j} f\right) \chi_{A}\right\|_{L}<\infty,
$$

then $f \in L$ and $f=h \circ T-h$ for some $h$ in $L$. This extends a recent result of [13]. We note that Orlicz spaces and Lorentz spaces are typical examples of such Banach lattices (see e.g. [8], [9]). In the next section we prove some auxiliary results.

2. Preliminaries. Let $(\Omega, \mathcal{A}, \mu), T$ and $f$ be as in the introduction. Let $\partial D$ be the boundary of the open unit disc $D$ in the complex plane, i.e., $\partial D=\left\{e^{i x}: 0 \leq x<2 \pi\right\}$, and $\mathcal{B}(\partial D)$ be the $\sigma$-field of all Borel subsets of $\partial D$. Denote by $d x$ the Lebesgue measure on $\partial D$. We consider the product measure space

$$
(\Omega \times \partial D, \mathcal{A} \otimes \mathcal{B}(\partial D), \mu \otimes d x) .
$$

For $s \in \mathbb{R}$, define an endomorphism $\tau_{s}$ of $(\Omega \times \partial D, \mathcal{A} \otimes \mathcal{B}(\partial D), \mu \otimes d x)$ by

$$
\tau_{s}\left(\omega, e^{i x}\right)=\left(T \omega, e^{i x} e^{-i s S_{1} f(\omega)}\right) .
$$


Since the function $g\left(\omega, e^{i x}\right)=e^{i x}$ is integrable on $(\Omega \times \partial D, \mathcal{A} \otimes \mathcal{B}(\partial D), \mu \otimes d x)$ and since $\tau_{s}^{j}\left(\omega, e^{i x}\right)=\left(T^{j} \omega, e^{i x} e^{-i s S_{j} f(\omega)}\right)$ for each $j \geq 1$, it follows from the Birkhoff ergodic theorem that the limit

$$
\lim _{n \rightarrow \infty} \frac{1}{n} \sum_{j=1}^{n} e^{i x} e^{-i s S_{j} f(\omega)}
$$

exists for $\mu \otimes d x$-a.e. $\left(\omega, e^{i x}\right) \in \Omega \times \partial D$. Then by Fubini's theorem the limit

$$
\lim _{n \rightarrow \infty} \frac{1}{n} \sum_{j=1}^{n} e^{-i s S_{j} f(\omega)}
$$

exists for $\mu$-a.e. $\omega \in \Omega$. We now define

$$
H(\omega, s)= \begin{cases}\lim _{n \rightarrow \infty} \frac{1}{n} \sum_{j=1}^{n} e^{-i s S_{j} f(\omega)} & \text { if the limit exists } \\ 2 & \text { otherwise. }\end{cases}
$$

Then $H(\omega, s)$ is a real-valued measurable function on $\Omega \times \mathbb{R}$ with respect to the $\sigma$-field $\mathcal{A} \otimes \mathcal{B}(\mathbb{R})$, where $\mathcal{B}(\mathbb{R})$ denotes the $\sigma$-field of all Borel subsets of $\mathbb{R}$. Since

$$
e^{-i s S_{1} f(\omega)} e^{-i s S_{j} f(T \omega)}=e^{-i s S_{j+1} f(\omega)}
$$

by the cocycle identity, it then follows that

$$
H(T \omega, s)=e^{i s S_{1} f(\omega)} H(\omega, s) \quad \text { whenever } H(\omega, s) \neq 2 .
$$

On the other hand, Fubini's theorem shows that the set

$$
\Omega_{1}=\{\omega \in \Omega: H(\omega, s) \neq 2 \text { for } d s \text {-a.e. } s \in \mathbb{R}\}
$$

is in $\mathcal{A}$. Furthermore,

$$
\mu\left(\Omega_{1}\right)=1 \quad \text { and } \quad T^{-1} \Omega_{1}=\Omega_{1} .
$$

Now, fix $\omega \in \Omega_{1}$. As a function of $s \in \mathbb{R}, H(\omega, s)$ is the $d s$-a.e. limit of the continuous positive definite functions $(1 / n) \sum_{j=1}^{n} \exp \left(-i s S_{j} f(\omega)\right)$, so there is a nonnegative finite Borel measure $\mu_{\omega}$ on $\mathbb{R}$ such that

$$
H(\omega, s)=\int_{\mathbb{R}} e^{i s t} d \mu_{\omega}(t) \quad \text { for } d s \text {-a.e. } s \in \mathbb{R}
$$

(cf. e.g. $\S 32$ and $\S 33$ of [7]). (This argument is due to Helson [5].) By (3) and the continuity of the mapping $\mathbb{R} \ni s \mapsto \int_{\mathbb{R}} e^{i s t} d \mu_{\omega}(t)$, we see that

$$
\int_{\mathbb{R}} e^{i s\left(S_{1} f(\omega)+t\right)} d \mu_{\omega}(t)=e^{i s S_{1} f(\omega)} \int_{\mathbb{R}} e^{i s t} d \mu_{\omega}(t)=\int_{\mathbb{R}} e^{i s t} d \mu_{T \omega}(t)
$$

for all $s \in \mathbb{R}$. Therefore

$$
\mu_{T \omega}(E)=\mu_{\omega}\left(E-S_{1} f(\omega)\right) \quad \text { for every } \omega \in \Omega_{1} \text { and } E \in \mathcal{B}(\mathbb{R}) .
$$


Next, let $N \geq 1$. For $\omega \in \Omega$ we put

$$
\begin{aligned}
& g_{N}(\omega)=\limsup _{n \rightarrow \infty} \frac{1}{n} \sum_{j=1}^{n} \chi_{[-N, N]}\left(S_{j} f(\omega)\right), \\
& g_{\infty}(\omega)=\lim _{N \rightarrow \infty} g_{N}(\omega) .
\end{aligned}
$$

Clearly, $g_{\infty}$ is measurable on $(\Omega, \mathcal{A}, \mu)$. Since $\left|S_{j} f(T \omega)-S_{j+1} f(\omega)\right|=$ $|f(\omega)|<\infty$, we observe that $g_{\infty}(\omega)>0$ if and only if $g_{\infty}(T \omega)>0$. Consequently, the set

$$
P=\left\{\omega \in \Omega: g_{\infty}(\omega)>0\right\} \cap \Omega_{1}
$$

belongs to $\mathcal{A}$, and $T^{-1} P=P$.

Suppose $\omega \in \Omega_{1}$. Since $\mu_{\omega}=0$ is equivalent to

$$
\int_{\mathbb{R}} \widehat{v}(t) d \mu_{\omega}(t)=0 \quad \text { for every } v \in L_{1}(\mathbb{R}, d s)
$$

and since

$$
\begin{aligned}
\int_{\mathbb{R}} \widehat{v}(t) d \mu_{\omega}(t) & =\int_{\mathbb{R} \mathbb{R}} v(s) e^{-i s t} d s d \mu_{\omega}(t) & & \\
& =\int_{\mathbb{R}}\left(v(s) \int_{\mathbb{R}} e^{-i s t} d \mu_{\omega}(t)\right) d s & & \text { (by Fubini's theorem) } \\
& =\int_{\mathbb{R}} v(s) H(\omega,-s) d s & & \text { (by }(6)) \\
& =\lim _{n \rightarrow \infty} \frac{1}{n} \sum_{j=1}^{n} \widehat{v}\left(-S_{j} f(\omega)\right) & & \text { (by }(2))
\end{aligned}
$$

it follows that $\mu_{\omega}=0$ is equivalent to

$$
\lim _{n \rightarrow \infty} \frac{1}{n} \sum_{j=1}^{n} \widehat{v}\left(-S_{j} f(\omega)\right)=0 \quad \text { for every } v \in L_{1}(\mathbb{R}, d s),
$$

which is clearly equivalent to $g_{\infty}(\omega)=0$. Thus

$$
P=\left\{\omega \in \Omega_{1}: \mu_{\omega}>0\right\} .
$$

The above argument shows that the function $P \ni \omega \mapsto \mu_{\omega}(\mathbb{R})$ is measurable with respect to the $\sigma$-field $\mathcal{A}$, and so is the function

$$
h(\omega)=\sup \left\{t \in \mathbb{R}: \mu_{\omega}((-\infty, t]) \leq \mu_{\omega}(\mathbb{R}) / 2\right\} \quad(\omega \in P) .
$$

The relation $h(T \omega)=f(\omega)+h(\omega)$ holds for every $\omega \in P$, by $(7)$.

We similarly see that the set

$$
P_{1}=\left\{\omega \in P: \mu_{\omega}(\mathbb{R})=1\right\}
$$

belongs to $\mathcal{A}$, and $T^{-1} P_{1}=P_{1}$ by $(7)$. 
Lastly, let $A \in \mathcal{A}$ be such that $A \subset \Omega_{1}, T^{-1} A=A$, and there exists a real-valued measurable function $h_{A}$ on $A$ satisfying

$$
f(\omega)=h_{A}(T \omega)-h_{A}(\omega) \quad \text { for every } \omega \in A .
$$

If we put

$$
\begin{aligned}
& A_{N}=\left\{\omega \in A:\left|h_{A}(\omega)\right| \leq N\right\} \quad(N \geq 1), \\
& \widetilde{g}_{N}(\omega)=\limsup _{n \rightarrow \infty} \frac{1}{n} \sum_{j=1}^{n} \chi_{A_{N}}\left(T^{j} \omega\right) \quad(\omega \in A),
\end{aligned}
$$

then the set

$$
\widetilde{A}=\left\{\omega \in A: \lim _{N \rightarrow \infty} \widetilde{g}_{N}(\omega)=1\right\}
$$

satisfies $\mu(\widetilde{A})=\mu(A)$ and $T^{-1} \widetilde{A}=\widetilde{A}$, by the Birkhoff ergodic theorem.

Suppose $\omega \in \widetilde{A}$. If $N \geq\left|h_{A}(\omega)\right|$, then

$$
\left|S_{j} f(\omega)\right|=\left|h_{A}\left(T^{j} \omega\right)-h_{A}(\omega)\right| \leq\left|h_{A}\left(T^{j} \omega\right)\right|+N,
$$

so that $T^{j} \omega \in A_{N}$ implies $\left|S_{j} f(\omega)\right| \leq 2 N$, and hence

$$
\frac{1}{n} \sum_{j=1}^{n} \chi_{A_{N}}\left(T^{j} \omega\right) \leq \frac{1}{n} \sum_{j=1}^{n} \chi_{[-2 N, 2 N]}\left(S_{j} f(\omega)\right) .
$$

On the other hand,

$$
\int_{\mathbb{R}} e^{i s t} d \mu_{\omega}(t)=\lim _{n \rightarrow \infty} \frac{1}{n} \sum_{j=1}^{n} e^{-i s S_{j} f(\omega)} \quad \text { for } d s \text {-a.e. } s \in \mathbb{R}
$$

by (2) and (6), since $\omega \in \widetilde{A} \subset A \subset \Omega_{1}$. Therefore we find that for $d s$-a.e. $s \in \mathbb{R}$ with $s=\theta / 2 N$ and $0<\theta \leq \pi / 4$,

$$
\begin{aligned}
\left|\int_{\mathbb{R}} e^{i s t} d \mu_{\omega}(t)\right| & =\lim _{n \rightarrow \infty} \frac{1}{n}\left|\sum_{j=1}^{n} e^{-i s S_{j} f(\omega)}\right| \\
& \geq \cos \theta \cdot\left(\limsup _{n \rightarrow \infty} \frac{1}{n} \sum_{j=1}^{n} \chi_{[-2 N, 2 N]}\left(S_{j} f(\omega)\right)\right)-\left(1-\widetilde{g}_{N}(\omega)\right) \\
& \geq \cos \theta \cdot \widetilde{g}_{N}(\omega)-\left(1-\widetilde{g}_{N}(\omega)\right) .
\end{aligned}
$$

Since $\widetilde{g}_{N}(\omega) \rightarrow 1$ as $N \rightarrow \infty$ and $\cos \theta \rightarrow 1$ as $\theta \rightarrow 0+0$, we must have $\mu_{\omega}(\mathbb{R}) \geq 1$. But this implies $\mu_{\omega}(\mathbb{R})=1$, because $\mu_{\omega}(\mathbb{R}) \leq 1$ is a direct consequence of $(6)$ and the fact that $|H(\omega, s)| \leq 1$ for $d s$-a.e. $s \in \mathbb{R}$. We have proved that $\widetilde{A} \subset P_{1}$.

We can summarize the above as follows.

FACT 1 (cf. Theorem 3 of [5]). Let $T$ be an endomorphism and $f$ be a real-valued measurable function on $\Omega$. Then the following hold: 
(I) The set $P_{1}=\left\{\omega \in \Omega_{1}: \mu_{\omega}(\mathbb{R})=1\right\}$ is a T-invariant measurable subset of $\Omega$, and there exists a real-valued measurable function $h$ on $P_{1}$ such that $f(\omega)=h(T \omega)-h(\omega)$ for every $\omega \in P_{1}$.

(II) $\mu_{\omega}=0$ for $\mu$-a.e. $\omega \in \Omega \backslash P_{1}$.

(III) If $A$ is a T-invariant measurable subset of $\Omega$ for which there exists a real-valued measurable function $h_{A}$ on $A$ such that $f(\omega)=h_{A}(T \omega)-h_{A}(\omega)$ for every $\omega \in A$, then $A \subset P_{1}(\bmod \mu)$, i.e., $\mu\left(A \backslash P_{1}\right)=0$.

FACT 2. Assume that $T$ is an ergodic endomorphism. Then there exists a real-valued measurable function $h$ on $\Omega$ such that $f(\omega)=h(T \omega)-h(\omega)$ for $\mu$-a.e. $\omega \in \Omega$ if and only if $\mu\left(\left\{\omega: g_{\infty}(\omega)>0\right\}\right)>0$.

Next we consider the probability measure space

$$
\left(K_{\mathbb{R}}, \mathcal{A}_{\mathbb{R}}, \mu_{\mathbb{R}}\right)=\left(\Omega \times \mathbb{R}, \mathcal{A} \otimes \mathcal{B}(\mathbb{R}), \mu \otimes \frac{d x}{\pi\left(1+x^{2}\right)}\right),
$$

and the null-preserving transformation $\vartheta$ of $\left(K_{\mathbb{R}}, \mathcal{A}_{\mathbb{R}}, \mu_{\mathbb{R}}\right)$ defined by

$$
\vartheta(\omega, x)=\left(T \omega, x+S_{1} f(\omega)\right) \quad \text { for }(\omega, x) \in K_{\mathbb{R}} .
$$

It follows that $\vartheta^{j}(\omega, x)=\left(T^{j} \omega, x+S_{j} f(\omega)\right)$ for $j \geq 0$.

Let $M:[0, \infty) \rightarrow[0, \infty)$ be an increasing function such that $\lim _{x \rightarrow \infty} M(x)$ $=\infty$ and there exist constants $k>0$ and $x_{0} \geq 0$ satisfying

$$
M(2 x) \leq k M(x) \quad \text { for } x \geq x_{0} .
$$

(See [9] for the properties of functions satisfying (17).)

Suppose $h$ is a real-valued measurable function on $\Omega$ such that $f(\omega)=$ $h(T \omega)-h(\omega)$ for $\mu$-a.e. $\omega \in \Omega$. Then define a function $w_{h}$ on $K_{\mathbb{R}}$ by

$$
w_{h}(\omega, x)=\frac{1+x^{2}}{1+(x-h(\omega))^{2}} \quad \text { for }(\omega, x) \in K_{\mathbb{R}} .
$$

It follows that $\int_{K_{\mathbb{R}}} w_{h} d \mu_{\mathbb{R}}=1$, and thus we can define a $\mu_{\mathbb{R}}$-equivalent probability measure $\lambda_{h}$ on $\left(K_{\mathbb{R}}, \mathcal{A}_{\mathbb{R}}\right)$ by

$$
\lambda_{h}=w_{h} d \mu_{\mathbb{R}} .
$$

We will prove that $\lambda_{h}$ is invariant with respect to $\vartheta$. To do this it may be assumed from the start that $f(\omega)=h(T \omega)-h(\omega)$ for every $\omega \in \Omega$. For $A \in \mathcal{A}$ and $\alpha, \beta \in \mathbb{R}$ with $\alpha<\beta$, we introduce a set $E$ in $\mathcal{A}_{\mathbb{R}}$ by

$$
E=\{(\omega, x): \omega \in A, h(\omega)+\alpha \leq x<h(\omega)+\beta\} .
$$

Then

$$
\begin{aligned}
\vartheta^{-1} E & =\{(\omega, x):(T \omega, x+f(\omega)) \in E\} \\
& =\left\{(\omega, x): \omega \in T^{-1} A, h(T \omega)+\alpha \leq x+f(\omega)<h(T \omega)+\beta\right\} \\
& =\left\{(\omega, x): \omega \in T^{-1} A, h(\omega)+\alpha \leq x<h(\omega)+\beta\right\},
\end{aligned}
$$


and thus the definition of $\lambda_{h}$ yields

$$
\lambda_{h}\left(\vartheta^{-1} E\right)=\lambda_{h}(E)
$$

since $T$ is an endomorphism of $(\Omega, \mathcal{A}, \mu)$. It follows from a standard approximation argument that $\lambda_{h}$ is invariant with respect to $\vartheta$.

On the other hand, an elementary calculation shows that

$$
\frac{1+x^{2}}{1+(x-t)^{2}}<2+t^{2} \quad \text { for } x, t \in \mathbb{R}
$$

whence

$$
0<w_{h}(\omega, x)<2+h^{2}(\omega) \quad \text { for all }(\omega, x) \in K_{\mathbb{R}} .
$$

Therefore, if $h$ is a function in $L_{\infty}(\Omega, \mu)$, then $w_{h}$ is a function in $L_{\infty}\left(K_{\mathbb{R}}, \mu_{\mathbb{R}}\right)$ such that $\left\|w_{h}\right\|_{\infty} \leq 2+\|h\|^{2}$.

Next, assume that $h \in L_{M}(\Omega, \mu)$, where $L_{M}(\Omega, \mu)$ denotes the space of all real-valued measurable functions $u$ on $(\Omega, \mathcal{A}, \mu)$ such that

$$
\int_{\Omega} M(|u(\omega)|) d \mu<\infty .
$$

Then we have, using Fubini's theorem,

$$
\begin{aligned}
\int_{K_{\mathbb{R}}} w_{h} M\left(\sqrt{w_{h}}\right) d \mu_{\mathbb{R}} & \leq \int_{\Omega} M\left(\sqrt{2+h^{2}(\omega)}\right)\left(\int_{\mathbb{R}} w_{h}(\omega, x) \frac{d x}{\pi\left(1+x^{2}\right)}\right) d \mu(\omega) \\
& =\int_{\Omega} M\left(\sqrt{2+h^{2}(\omega)}\right) d \mu(\omega) \leq \int_{\Omega} M(2+|h(\omega)|) d \mu<\infty,
\end{aligned}
$$

where the last inequality comes from (17). Consequently, $h \in L_{M}(\Omega, \mu)$ implies that the function $w_{h} M\left(\sqrt{w_{h}}\right)$ belongs to $L_{1}\left(K_{\mathbb{R}}, \mu_{\mathbb{R}}\right)$.

Conversely, assume that $\lambda=w d \mu_{\mathbb{R}}$, where $0 \leq w \in L_{1}\left(K_{\mathbb{R}}, \mu_{\mathbb{R}}\right)$, is a $\mu_{\mathbb{R}}$-absolutely continuous probability measure invariant with respect to $\vartheta$. Then we introduce a function $w_{\Omega}$ on $\Omega$ by

$$
w_{\Omega}(\omega)=\int_{\mathbb{R}} w(\omega, x) \frac{d x}{\pi\left(1+x^{2}\right)} \quad(\omega \in \Omega) .
$$

Fubini's theorem implies that $w_{\Omega} \in L_{1}(\Omega, \mu)$, and thus $\lambda_{\Omega}=w_{\Omega} d \mu$ is a $\mu$-absolutely continuous probability measure invariant with respect to $T$. Notice that if $T$ is assumed to be ergodic, then $w_{\Omega}(\omega)=1$ for $\mu$-a.e. $\omega \in \Omega$. On the other hand, if $\lambda$ is a $\mu_{\mathbb{R}}$-equivalent probability measure, then $w_{\Omega}(\omega)>0$ for $\mu$-a.e. $\omega \in \Omega$, without assuming the ergodicity of $T$.

Thus, in the following, we will assume that $w_{\Omega}(\omega)>0$ for $\mu$-a.e. $\omega \in \Omega$. Then we can define a Borel probability measure $\lambda_{\omega}$ on $\mathbb{R}$, for $\mu$-a.e. $\omega \in \Omega$, by

$$
\lambda_{\omega}(B)=\frac{1}{w_{\Omega}(\omega)} \int_{B} w(\omega, x) \frac{d x}{\pi\left(1+x^{2}\right)} \quad(B \in \mathcal{B}(\mathbb{R})) .
$$


To prove the existence of a real-valued measurable function $h$ on $\Omega$ such that $f(\omega)=h(T \omega)-h(\omega)$ for $\mu$-a.e. $\omega \in \Omega$, we must assume below that $T$ is an automorphism. By this assumption, both $\vartheta$ and $\vartheta^{-1}$ are null-preserving transformations of $\left(K_{\mathbb{R}}, \mathcal{A}_{\mathbb{R}}, \mu_{\mathbb{R}}\right)$; and if $A \in \mathcal{A}$ and $B \in \mathcal{B}(\mathbb{R})$, then

$$
\lambda(A \times B)=\int_{A} \lambda_{\omega}(B) w_{\Omega}(\omega) d \mu(\omega),
$$

and

$$
\begin{aligned}
\lambda(\vartheta(A \times B)) & =\int_{T A} \lambda_{\omega}\left(B+f\left(T^{-1} \omega\right)\right) w_{\Omega}(\omega) d \mu(\omega) \\
& =\int_{A} \lambda_{T \omega}(B+f(\omega)) w_{\Omega}(\omega) d \mu(\omega),
\end{aligned}
$$

where the last equality comes from the invariance of the measure $\lambda_{\Omega}=$ $w_{\Omega} d \mu$ with respect to $T$. Since $\lambda(A \times B)=\lambda(\vartheta(A \times B))$, it follows that $\lambda_{\omega}(B)=\lambda_{T \omega}(B+f(\omega))$ for $\mu$-a.e. $\omega \in \Omega$, and since $\mathcal{B}(\mathbb{R})$ is separable, this shows that $\lambda_{\omega}(B)=\lambda_{T \omega}(B+f(\omega))$ for all $B \in \mathcal{B}(\mathbb{R})$ and for $\mu$-a.e. $\omega \in \Omega$. Consequently, the function

$$
h(\omega)=\sup \left\{t \in \mathbb{R}: \lambda_{\omega}((-\infty, t])=1 / 2\right\} \quad(\omega \in \Omega)
$$

satisfies $h(T \omega)=h(\omega)+f(\omega)$ for $\mu$-a.e. $\omega \in \Omega$, and is measurable with respect to $\mathcal{A}$ by an easy approximation argument.

Now, assume that $w=d \lambda / d \mu_{\mathbb{R}} \in L_{\infty}\left(K_{\mathbb{R}}, \mu_{\mathbb{R}}\right)$. Since $\lambda$ is invariant with respect to $\vartheta$ by hypothesis, it follows that the set

$$
E_{\lambda}=\left\{(\omega, x) \in K_{\mathbb{R}}: w(\omega, x)>0\right\}
$$

satisfies $\vartheta^{-1} E_{\lambda}=E_{\lambda}\left(\bmod \mu_{\mathbb{R}}\right)$. On the other hand, the measure $\lambda_{h}=$ $w_{h} d \mu_{\mathbb{R}}$ is also invariant with respect to $\vartheta$ (cf. (18)-(20)). Thus it follows from the Neveu-Chacon identification theorem (see e.g. Theorem 3.3.4 of [10]) for the Chacon-Ornstein ratio ergodic limit that the function $w / w_{h}$ is measurable with respect to the $\sigma$-field

$$
\mathcal{I}_{\mathbb{R}}=\left\{A \in \mathcal{A}_{\mathbb{R}}: \vartheta^{-1} A=A\left(\bmod \mu_{\mathbb{R}}\right)\right\} .
$$

Thus there exists a real-valued positive function $G$ on $E_{\lambda}$, measurable with respect to $\mathcal{I}_{\mathbb{R}}$, such that

$$
w_{h}=G \cdot w\left(\bmod \mu_{\mathbb{R}}\right) \quad \text { on } E_{\lambda} .
$$

Since $G \circ \vartheta=G\left(\bmod \mu_{\mathbb{R}}\right)$ on $E_{\lambda}$, it follows that for $\mu_{\mathbb{R}}$-a.e. $(\omega, x) \in E_{\lambda}$,

$$
\begin{aligned}
\sup \left\{w_{h}\left(\vartheta^{j}(\omega, x)\right): j \geq 0\right\} & =G(\omega, x) \cdot \sup \left\{w\left(\vartheta^{j}(\omega, x)\right): j \geq 0\right\} \\
& \leq G(\omega, x) \cdot\|w\|_{\infty} .
\end{aligned}
$$


The definition of $w_{h}$ (cf. (18)) implies that for $\mu_{\mathbb{R}}$-a.e. $(\omega, x) \in K_{\mathbb{R}}$,

$$
\begin{aligned}
w_{h}\left(\vartheta^{j}(\omega, x)\right) & =w_{h}\left(T^{j} \omega, x+S_{j} f(\omega)\right)=w_{h}\left(T^{j} \omega, x+h\left(T^{j} \omega\right)-h(\omega)\right) \\
& =\frac{1+\left(x+h\left(T^{j} \omega\right)-h(\omega)\right)^{2}}{1+(x-h(\omega))^{2}} .
\end{aligned}
$$

Hence

$$
\sup _{j \geq 0} \frac{1+\left(x+h\left(T^{j} \omega\right)-h(\omega)\right)^{2}}{1+(x-h(\omega))^{2}}<\infty \quad \text { for } \mu_{\mathbb{R}} \text {-a.e. }(\omega, x) \in E_{\lambda},
$$

and therefore the function

$$
g(\omega)=\sup _{j \geq 0}\left|h\left(T^{j} \omega\right)\right| \quad(\omega \in \Omega)
$$

satisfies $g(\omega)<\infty$ for $\mu_{\mathbb{R}}$-a.e. $(\omega, x) \in E_{\lambda}$. Now, if $\lambda$ is a $\mu_{\mathbb{R}}$-equivalent measure, then, since $E_{\lambda}=K_{\mathbb{R}}\left(\bmod \mu_{\mathbb{R}}\right)$, it follows that $g(\omega)<\infty$ for $\mu$-a.e. $\omega \in \Omega$. If $T$ is ergodic, and $\lambda$ is a $\mu_{\mathbb{R}}$-absolutely continuous probability measure, then, since $\mu_{\mathbb{R}}\left(E_{\lambda}\right)>0$, the set $\{\omega \in \Omega: g(\omega)<\infty\}$ is of positive $\mu$-measure, and the ergodicity of $T$ implies that $g(\omega)<\infty$ for $\mu$-a.e. $\omega \in \Omega$. Thus, in either case, $0 \leq g(T \omega) \leq g(\omega)<\infty$ for $\mu$-a.e. $\omega \in \Omega$. Since $\mu$ is an invariant measure with respect to $T$, it then follows that $g(T \omega)=g(\omega)$ for $\mu$-a.e. $\omega \in \Omega$, and hence the sets

$$
A_{n}=\{\omega \in \Omega: n-1 \leq g(\omega)<n\}, \quad n \geq 1,
$$

satisfy $T^{-1} A_{n}=A_{n}(\bmod \mu)$ and $\Omega=\bigcup_{n=1}^{\infty} A_{n}(\bmod \mu)$. In particular, if $T$ is ergodic, then $g$ is a constant function in $L_{\infty}(\Omega, \mu)$.

Next, assume that the function $w=d \lambda / d \mu_{\mathbb{R}}$ is such that $w M(\sqrt{w}) \in$ $L_{1}\left(K_{\mathbb{R}}, \mu_{\mathbb{R}}\right)$. Let $v_{j}$ denote the Radon-Nikodym derivative $d\left(\mu_{\mathbb{R}} \circ \vartheta^{j}\right) / d \mu_{\mathbb{R}}$, where $\mu_{\mathbb{R}} \circ \vartheta^{j}$ denotes the measure defined by $\left(\mu_{\mathbb{R}} \circ \vartheta^{j}\right)(A)=\mu_{\mathbb{R}}\left(\vartheta^{j} A\right)$ for $A \in \mathcal{A}_{\mathbb{R}}$. Since $\vartheta^{j}(\omega, x)=\left(T^{j} \omega, x+S_{j} f(\omega)\right)$ for $(\omega, x) \in K_{\mathbb{R}}$, it is easy to see that $v_{j}$ has the form

$$
\begin{aligned}
v_{j}(\omega, x) & =\frac{1+x^{2}}{1+\left(x+S_{j} f(\omega)\right)^{2}} \\
& =\frac{1+x^{2}}{1+\left(x+h\left(T^{j} \omega\right)-h(\omega)\right)^{2}} \quad \text { for }(\omega, x) \in K_{\mathbb{R}} .
\end{aligned}
$$

Since $\lambda=w d \mu_{\mathbb{R}}$ is invariant with respect to $\vartheta$ by hypothesis, it follows that if $A \in \mathcal{A}_{\mathbb{R}}$, then

$$
\int_{A} w d \mu_{\mathbb{R}}=\int_{\vartheta^{j} A} w d \mu_{\mathbb{R}}=\int\left(w \cdot \chi_{\vartheta^{j} A}\right) \circ \vartheta^{j} d\left(\mu_{\mathbb{R}} \circ \vartheta^{j}\right)=\int_{A}\left(w \circ \vartheta^{j}\right) v_{j} d \mu_{\mathbb{R}},
$$

whence $w=\left(w \circ \vartheta^{j}\right) v_{j}\left(\bmod \mu_{\mathbb{R}}\right)$ on $K_{\mathbb{R}}$. Therefore,

$$
w^{1 / 2}(\omega, x) \frac{\left|x+h\left(T^{j} \omega\right)-h(\omega)\right|}{\sqrt{1+x^{2}}} \leq w^{1 / 2} \circ \vartheta^{j}(\omega, x)
$$


for $\mu_{\mathbb{R}}$-a.e. $(\omega, x) \in K_{\mathbb{R}}$. Now, if $C \cdot w^{1 / 2}(\omega, x) \geq \sqrt{1+x^{2}}$ for some constant $C>1$, then

$$
\left|x+h\left(T^{j} \omega\right)-h(\omega)\right| \leq C \cdot w^{1 / 2} \circ \vartheta^{j}(\omega, x)
$$

and by (17) there exist constants $k(C)>0$ and $x_{1} \geq 0$ such that

$$
M\left(\left|x+h\left(T^{j} \omega\right)-h(\omega)\right|\right) \leq k(C) \cdot M\left(w^{1 / 2} \circ \vartheta^{j}(\omega, x)\right)
$$

whenever $w^{1 / 2} \circ \vartheta^{j}(\omega, x) \geq x_{1}$; consequently,

$$
\begin{aligned}
\limsup _{n \rightarrow \infty} & \frac{1}{n} \sum_{j=1}^{n} M\left(\left|x+h\left(T^{j} \omega\right)-h(\omega)\right|\right) \\
\leq & M\left(C x_{1}\right)+k(C) \cdot \limsup _{n \rightarrow \infty} \frac{1}{n} \sum_{j=1}^{n} M\left(w^{1 / 2} \circ \vartheta^{j}(\omega, x)\right) \\
& =M\left(C x_{1}\right)+k(C) \cdot E\left\{M\left(w^{1 / 2}\right) \mid\left(K_{\mathbb{R}}, \mathcal{I}_{\mathbb{R}}, w d \mu_{\mathbb{R}}\right)\right\}(\omega, x)<\infty
\end{aligned}
$$

by the Birkhoff ergodic theorem, where $E\left\{M\left(w^{1 / 2}\right) \mid\left(K_{\mathbb{R}}, \mathcal{I}_{\mathbb{R}}, w d \mu_{\mathbb{R}}\right)\right\}$ denotes the conditional expectation of the function $M\left(w^{1 / 2}\right)$ with respect to the $\sigma$-field $\mathcal{I}_{\mathbb{R}}$ and the measure $w d \mu_{\mathbb{R}}$. Since the constant $C>0$ can be arbitrarily large, this proves that

$$
\limsup _{n \rightarrow \infty} \frac{1}{n} \sum_{j=1}^{n} M\left(\left|x+h\left(T^{j} \omega\right)-h(\omega)\right|\right)<\infty \quad \text { for } \mu_{\mathbb{R}^{-}} \text {a.e. }(\omega, x) \in E_{\lambda} .
$$

Hence, if we define a function $\widetilde{g}$ on $\Omega$ by

$$
\widetilde{g}(\omega)=\limsup _{n \rightarrow \infty} \frac{1}{n} \sum_{j=1}^{n} M\left(\left|h\left(T^{j} \omega\right)\right|\right) \quad \text { for } \omega \in \Omega,
$$

then (29) and (17) show that $\widetilde{g}(\omega)<\infty$ for $\mu_{\mathbb{R}}$-a.e. $(\omega, x) \in E_{\lambda}$. Therefore, as before, if $\lambda$ is a $\mu_{\mathbb{R}}$-equivalent measure, or if $T$ is ergodic and $\lambda$ is a $\mu_{\mathbb{R}}$-absolutely continuous probability measure, then $0 \leq \widetilde{g}(T \omega)=\widetilde{g}(\omega)<\infty$ for $\mu$-a.e. $\omega \in \Omega$; and the sets $B_{n}=\{\omega \in \Omega: n-1 \leq \widetilde{g}(\omega)<n\}, n \geq 1$, satisfy $T^{-1} B_{n}=B_{n}, \int_{B_{n}} M(|h|) d \mu=\int_{B_{n}} \widetilde{g} d \mu<\infty$ (by the Birkhoff ergodic theorem), and $\Omega=\bigcup_{n=1}^{\infty} B_{n}(\bmod \mu)$. In particular, if $T$ is ergodic, then $\widetilde{g}(\omega)=\int_{\Omega} M(|h|) d \mu<\infty$ for $\mu$-a.e. $\omega \in \Omega$.

We can summarize the above as follows.

FACT 3 (cf. Proposition 3.1 of [1]). Let $M:[0, \infty) \rightarrow[0, \infty)$ be an increasing function such that $\lim _{x \rightarrow \infty} M(x)=\infty$ and there exist constants $k>0$ and $x_{0} \geq 0$ satisfying (17). Then the following hold:

(I) If $T$ is an endomorphism and $h$ is a real-valued measurable function on $\Omega$ such that $f(\omega)=h(T \omega)-h(\omega)$ for $\mu$-a.e. $\omega \in \Omega$, then the measure $\lambda_{h}=w_{h} d \mu_{\mathbb{R}}$ in (19) is a $\mu_{\mathbb{R}}$-equivalent probability measure invariant with 
respect to $\vartheta$. If moreover $h \in L_{\infty}(\Omega, \mu)$ then $w_{h} \in L_{\infty}\left(K_{\mathbb{R}}, \mu_{\mathbb{R}}\right)$; and if $h \in L_{M}(\Omega, \mu)$ then $w_{h} M\left(\sqrt{w_{h}}\right) \in L_{1}\left(K_{\mathbb{R}}, \mu_{\mathbb{R}}\right)$.

(II) If $T$ is an automorphism and $\lambda=w d \mu_{\mathbb{R}}$, where $0<w \in L_{1}\left(K_{\mathbb{R}}, \mu_{\mathbb{R}}\right)$, is a $\mu_{\mathbb{R}}$-equivalent probability measure invariant with respect to $\vartheta$, then there exists a real-valued measurable function $h$ on $\Omega$ such that $f(\omega)=$ $h(T \omega)-h(\omega)$ for $\mu$-a.e. $\omega \in \Omega$. If moreover $w \in L_{\infty}\left(K_{\mathbb{R}}, \mu_{\mathbb{R}}\right)[$ resp. $\left.w M(\sqrt{w}) \in L_{1}\left(K_{\mathbb{R}}, \mu_{\mathbb{R}}\right)\right]$, then there exists a countable measurable decomposition $\left\{A_{n}: n \geq 1\right\}$ of $\Omega$ with $T^{-1} A_{n}=A_{n}$ for $n \geq 1$ such that $h \chi_{A_{n}} \in L_{\infty}(\Omega, \mu)\left[\right.$ resp. $\left.h \chi_{A_{n}} \in L_{M}(\Omega, \mu)\right]$ for every $n \geq 1$.

(III) If $T$ is an ergodic automorphism and $\lambda=w d \mu_{\mathbb{R}}$, where $0 \leq w \in$ $L_{1}\left(K_{\mathbb{R}}, \mu_{\mathbb{R}}\right)$, is a $\mu_{\mathbb{R}}$-absolutely continuous probability measure invariant with respect to $\vartheta$, then there exists a real-valued measurable function $h$ on $\Omega$ such that $f(\omega)=h(T \omega)-h(\omega)$ for $\mu$-a.e. $\omega \in \Omega$. If moreover $w \in L_{\infty}\left(K_{\mathbb{R}}, \mu_{\mathbb{R}}\right)$ $\left[\right.$ resp. $\left.w M(\sqrt{w}) \in L_{1}\left(K_{\mathbb{R}}, \mu_{\mathbb{R}}\right)\right]$, then $h \in L_{\infty}(\Omega, \mu)\left[\right.$ resp. $\left.h \in L_{M}(\Omega, \mu)\right]$.

\section{Main results}

Theorem 1 (cf. Theorem 1 on p. 62 of [6]). Let $\varphi: \mathbb{R} \rightarrow[0, \infty)$ be a Borel measurable function on $\mathbb{R}$ such that $\lim _{|x| \rightarrow \infty} \varphi(x)=\infty$. Assume that $T$ is an ergodic endomorphism. If there exists a set $A \in \mathcal{A}$ with $\mu(A)>0$ such that

$$
\liminf _{n \rightarrow \infty} \frac{1}{n} \sum_{j=1}^{n} \int_{A} \varphi\left(S_{j} f(\omega)\right) d \mu<\infty,
$$

then there exists a real-valued measurable function $h$ on $\Omega$ such that $f(\omega)=$ $h(T \omega)-h(\omega)$ for $\mu$-a.e. $\omega \in \Omega$. If , in addition, $\sup \{\varphi(x):|x| \leq N\}<\infty$ for every $N \geq 1$, and $\lim \sup _{|x| \rightarrow \infty} \varphi(x+a) / \varphi(x)<\infty$ for every $a \in \mathbb{R}$, then $\int_{\Omega} \varphi(h(\omega)) d \mu<\infty$.

Corollary (cf. Theorem 3.2 of [1]). Let $T$ be an ergodic endomorphism, and $0<r<\infty$. If

$$
\liminf _{n \rightarrow \infty} \frac{1}{n} \sum_{j=1}^{n} \int_{A}\left|S_{j} f(\omega)\right|^{r} d \mu<\infty
$$

for some $A \in \mathcal{A}$ with $\mu(A)>0$, then there exists a real-valued measurable function $h$ on $\Omega$ with $\int_{\Omega}|h(\omega)|^{r} d \mu<\infty$ such that $f(\omega)=h(T \omega)-h(\omega)$ for $\mu$-a.e. $\omega \in \Omega$.

Proof of Theorem 1. By Fatou's lemma, the function

$$
\varphi(f)_{*}(\omega)=\liminf _{n \rightarrow \infty} \frac{1}{n} \sum_{j=1}^{n} \varphi\left(S_{j} f(\omega)\right)
$$


satisfies

$$
\int_{A} \varphi(f)_{*}(\omega) d \mu \leq \liminf _{n \rightarrow \infty} \frac{1}{n} \sum_{j=1}^{n} \int_{A} \varphi\left(S_{j} f(\omega)\right) d \mu<\infty,
$$

whence $\varphi(f)_{*}(\omega)<\infty$ for $\mu$-a.e. $\omega \in A$. Since $\lim _{|x| \rightarrow \infty} \varphi(x)=\infty$, the inequality $\varphi(f)_{*}(\omega)<\infty$ implies the existence of an $N \geq 1$ such that

$$
g_{N}(\omega)=\limsup _{n \rightarrow \infty} \frac{1}{n} \sum_{j=1}^{n} \chi_{[-N, N]}\left(S_{j} f(\omega)\right)>0 .
$$

It follows that $g_{\infty}(\omega)=\lim _{N \rightarrow \infty} g_{N}(\omega)>0$ for $\mu$-a.e. $\omega \in A$, and therefore by Fact 2 there exists a real-valued measurable function $h$ on $\Omega$ such that $f(\omega)=h(T \omega)-h(\omega)$ for $\mu$-a.e. $\omega \in \Omega$. Assume that $\varphi$ satisfies $\sup \{\varphi(x)$ : $|x| \leq N\}<\infty$ for every $N \geq 1$, and $\lim \sup _{|x| \rightarrow \infty} \varphi(x+a) / \varphi(x)<\infty$ for every $a \in \mathbb{R}$. Then, since $S_{j} f(\omega)=h\left(T^{j} \omega\right)-h(\omega)$ on $\Omega$, it follows that

$$
\liminf _{n \rightarrow \infty} \frac{1}{n} \sum_{j=1}^{n} \varphi\left(h\left(T^{j} \omega\right)\right)=\liminf _{n \rightarrow \infty} \frac{1}{n} \sum_{j=1}^{n} \varphi\left(S_{j} f(\omega)+h(\omega)\right)<\infty
$$

for $\mu$-a.e. $\omega \in A$ with $\varphi(f)_{*}(\omega)<\infty$, so that the function

$$
h_{*}(\omega)=\liminf _{n \rightarrow \infty} \frac{1}{n} \sum_{j=1}^{n} \varphi\left(h\left(T^{j} \omega\right)\right)
$$

satisfies $h_{*}(\omega)<\infty$ for $\mu$-a.e. $\omega \in A$. Since $h_{*}$ is an invariant function with respect to $T$, the Birkhoff ergodic theorem and the ergodicity of $T$ imply that $h_{*}(\omega)=\int_{\Omega} \varphi \circ h d \mu<\infty$ for $\mu$-a.e. $\omega \in \Omega$, and hence the desired result has been established.

REMARK 1. If the ergodicity of $T$ is not assumed in Theorem 1, then the above argument shows that inequality (30) with $A=\Omega$ implies the existence of a real-valued measurable function $h$ on $\Omega$ such that $f(\omega)=h(T \omega)-h(\omega)$ for $\mu$-a.e. $\omega \in \Omega$; if moreover $\sup \{\varphi(x):|x| \leq N\}<\infty$ for every $N \geq 1$, and $\lim \sup _{|x| \rightarrow \infty} \varphi(x+a) / \varphi(x)<\infty$ for every $a \in \mathbb{R}$, then there exists a countable measurable decomposition $\left\{A_{n}: n \geq 1\right\}$ of $\Omega$ with $T^{-1} A_{n}=A_{n}$ for $n \geq 1$ such that $\int_{A_{n}} \varphi(h(\omega)) d \mu<\infty$ for every $n \geq 1$.

From now on we consider a Banach lattice $\left(L,\|\cdot\|_{L}\right)$ of equivalence classes of real-valued measurable functions on $\Omega$. Thus, two functions $u$ and $v$ in $L$ are not distinguished provided that $u(\omega)=v(\omega)$ for $\mu$-a.e. $\omega \in \Omega$. By definition, the norm $\|\cdot\|_{L}$ has the property:

(A) If $u, v \in L$ and $|u(\omega)| \leq|v(\omega)|$ for $\mu$-a.e. $\omega \in \Omega$, then $\|u\|_{L} \leq\|v\|_{L}$. In this paper we assume the additional properties:

(B) If $v$ is a real-valued measurable function on $\Omega$ and there exists a function $u \in L$ such that $|v(\omega)| \leq|u(\omega)|$ for $\mu$-a.e. $\omega \in \Omega$, then $v \in L$. 
(C) If $\left(u_{n}\right)$ is a sequence of functions in $L$ such that $\left|u_{1}(\omega)\right| \leq$ $\left|u_{2}(\omega)\right| \leq \ldots$ for $\mu$-a.e. $\omega \in \Omega$, and $\sup _{n \geq 1}\left\|u_{n}\right\|_{L}<\infty$, then there exists a function $u \in L$ such that $\left|u_{n}(\omega)\right| \leq|u(\omega)|$ for $\mu$-a.e. $\omega \in \Omega$ and all $n \geq 1$.

(D) If $v$ is a real-valued measurable function on $\Omega$ and $u \in L$ is such that

$$
\mu(\{\omega:|v(\omega)|>a\})=\mu(\{\omega:|u(\omega)|>a\})
$$

for every $0<a \in \mathbb{R}$, then $v \in L$ and $\|v\|_{L}=\|u\|_{L}$.

It should be noted that, besides the usual $L_{p}(\Omega, \mu)$-spaces with $1 \leq p$ $\leq \infty$, there are many interesting Banach lattices of functions which share these additional properties (B), (C) and (D). Examples are Orlicz spaces, Lorentz spaces, etc. By Property (D), the mapping $u \mapsto u \circ T$ is a linear isometry of $\left(L,\|\cdot\|_{L}\right)$ for every endomorphism $T$.

THEOREM 2 (cf. [13]). Assume that $T$ is an ergodic endomorphism. If there exists a set $A \in \mathcal{A}$ with $\mu(A)>0$ such that $\left(S_{j} f\right) \chi_{A} \in L$ for $j \geq 1$ and

$$
K:=\liminf _{n \rightarrow \infty} \frac{1}{n} \sum_{j=1}^{n}\left\|\left(S_{j} f\right) \chi_{A}\right\|_{L}<\infty
$$

then there exists a function $h$ in $L$ such that $f=h \circ T-h$ on $\Omega$.

Proof. Define

$$
\begin{aligned}
f_{n}(\omega) & =\inf _{m \geq n} \frac{1}{m} \sum_{j=1}^{m}\left|S_{j} f(\omega)\right| \quad \text { for } n \geq 1, \\
f_{\infty}(\omega) & =\lim _{n \rightarrow \infty} f_{n}(\omega) .
\end{aligned}
$$

By Property (B) and the hypothesis $\left(S_{j} f\right) \chi_{A} \in L$ for $j \geq 1$ it follows that $f_{n} \chi_{A} \in L$ for $n \geq 1$; and by Property (A) we have $\left\|f_{n} \chi_{A}\right\|_{L} \leq$ $(1 / m) \sum_{j=1}^{m}\left\|\left(S_{j} f\right) \chi_{A}\right\|_{L}$ for $m \geq n$. Thus

$$
\left\|f_{n} \chi_{A}\right\|_{L} \leq \liminf _{m \rightarrow \infty} \frac{1}{m} \sum_{j=1}^{m}\left\|\left(S_{j} f\right) \chi_{A}\right\|_{L}=K \quad \text { for every } n \geq 1
$$

and hence

$$
f_{\infty} \chi_{A} \in L
$$

by Properties (C) and (B). In particular, $f_{\infty}(\omega)<\infty$ for $\mu$-a.e. $\omega \in A$, and thus we can apply the proof of Theorem 1 with $\varphi(x)=|x|$ to infer that there exists a real-valued measurable function $h$ on $\Omega$ with $\int_{\Omega}|h(\omega)| d \mu<\infty$ such that $f(\omega)=h(T \omega)-h(\omega)$ for $\mu$-a.e. $\omega \in \Omega$.

To prove that $h \in L$, we may assume below that $(\Omega, \mathcal{A}, \mu)$ is nonatomic. (Indeed, if $(\Omega, \mathcal{A}, \mu)$ is atomic, then the ergodicity of $T$ implies that $\Omega$ is essentially a finite set, and then $h \in L$ is obvious.) Further we may assume 
that $(\Omega, \mathcal{A}, \mu)$ is separable, because there is a countable family $\left\{A_{i}: i \geq 1\right\}$ in $\mathcal{A}$ such that

(i) the measure space $\left(\Omega, \mathcal{A}_{1}, \mu\right)$, where $\mathcal{A}_{1}$ denotes the $\sigma$-field generated by $\left\{A_{i}: i \geq 1\right\}$, is nonatomic,

(ii) $T$ is an ergodic endomorphism of $\left(\Omega, \mathcal{A}_{1}, \mu\right)$,

(iii) $f$ is measurable with respect to $\mathcal{A}_{1}$, and

(iv) the space $L_{1}=\left\{g \in L: g\right.$ is $\mathcal{A}_{1}$-measurable $\}$, with norm $\|\cdot\|_{L_{1}}=$ the restriction of the norm $\|\cdot\|_{L}$ to $L_{1}$, is a Banach lattice having Properties (B)-(D) with $L_{1}$ and $\|\cdot\|_{L_{1}}$ in place of $L$ and $\|\cdot\|_{L}$, respectively.

Next, we use the isomorphism theorem for measure algebras (cf. e.g. Theorem 41.C of [4]) to infer that there exists an isomorphism $\Phi$ from the measure algebra $(\mathcal{A}(\mu), \mu)$ onto the measure algebra $(\mathcal{B}([0,1]), d x)$, where $\mathcal{B}([0,1])$ denotes the $\sigma$-field of all Borel subsets of $[0,1]$ and $d x$ is the Lebesgue measure. Thus we may assume that $(\Omega, \mathcal{A}, \mu)=([0,1], \mathcal{B}([0,1]), d x)$. Then $T$ can be regarded as an isomorphism from $(\mathcal{B}([0,1]), d x)$ into itself. Since $[0,1]$ is an uncountable complete separable metric space, it then follows (see e.g. Proposition 15.19 of [12]) that $T$ can be considered to be an (ergodic) endomorphism of the measure space $([0,1], \mathcal{B}([0,1]), d x)$.

We then use the natural extension of the ergodic endomorphism $T$ of $([0,1], \mathcal{B}([0,1]), d x)$ (cf. e.g. $\S 4$ of Chapter 10 of $[3])$. That is, it is known that there exists an ergodic automorphism $\widetilde{T}$ of a separable nonatomic probability measure space $(\widetilde{\Omega}, \widetilde{\mathcal{A}}, \widetilde{\mu})$, and a measure preserving transformation $S$ from $(\widetilde{\Omega}, \widetilde{\mathcal{A}}, \widetilde{\mu})$ to $(\Omega, \mathcal{A}, \mu)$ such that

$$
(S \circ \widetilde{T})(\widetilde{\omega})=(T \circ S)(\widetilde{\omega}) \quad \text { for every } \widetilde{\omega} \in \widetilde{\Omega} .
$$

Denote by $\widetilde{L}$ the space of all real-valued $\widetilde{\mathcal{A}}$-measurable functions $\widetilde{u}$ on $\widetilde{\Omega}$ to which there corresponds a function $u \in L$ such that $\widetilde{\mu}(\{\widetilde{\omega}:|\widetilde{u}(\widetilde{\omega})|>a\})=$ $\mu(\{\omega:|u(\omega)|>a\})$ for every $0<a \in \mathbb{R}$, and define $\|\widetilde{u}\|_{\widetilde{L}}=\|u\|_{L}$. By using Property (D) it is easy to check that if $\widetilde{u}$ and $\widetilde{v}$ are in $\widetilde{L}$, then $\widetilde{u}+\widetilde{v} \in \widetilde{L}$ and $\|\widetilde{u}+\widetilde{v}\|_{\widetilde{L}} \leq\|\widetilde{u}\|_{\widetilde{L}}+\|\widetilde{v}\|_{\widetilde{L}}$. (In fact, by the isomorphism theorem, there exists an isomorphism $\Psi$ from the measure algebra $(\widetilde{\mathcal{A}}(\widetilde{\mu}), \widetilde{\mu})$ onto $(\mathcal{A}(\mu), \mu)$. Then $\Psi$ can be uniquely extended, in an obvious manner, to an invertible linear operator (denoted also by $\Psi$ ) from $L_{0}(\widetilde{\Omega}, \widetilde{\mu})$ onto $L_{0}(\Omega, \mu)$, where $L_{0}(\widetilde{\Omega}, \widetilde{\mu})$ is the space of all real-valued measurable functions on $(\widetilde{\Omega}, \widetilde{\mathcal{A}}, \widetilde{\mu})$ and, by definition, two functions $\widetilde{u}$ and $\widetilde{v}$ in $L_{0}(\widetilde{\Omega}, \widetilde{\mu})$ are not distinguished provided that $\widetilde{u}(\widetilde{\omega})=\widetilde{v}(\widetilde{\omega})$ for $\widetilde{\mu}$-a.e. $\widetilde{\omega} \in \widetilde{\Omega} ; L_{0}(\Omega, \mu)$ is defined similarly for $(\Omega, \mathcal{A}, \mu)$. Then $\Psi \widetilde{L}=L$ and $\|\widetilde{u}\|_{\widetilde{L}}=\|\Psi \widetilde{u}\|_{L}$ for all $\widetilde{u} \in \widetilde{L}$. $)$ It follows that $\left(\widetilde{L},\|\cdot\|_{\widetilde{L}}\right)$ is a Banach lattice having Properties (B)-(D) with $\widetilde{L}$ and $\|\cdot\|_{\widetilde{L}}$ replaced by $L$ and $\|\cdot\|_{L}$, respectively. In order to prove that $h \in L$, it is now sufficient to prove that $h \circ S \in \widetilde{L}$. 
Consequently, we have reached the conclusion that $T$ may be assumed to be an ergodic automorphism of $(\Omega, \mathcal{A}, \mu)$. With this assumption we continue the proof as follows.

First, as is easily seen, it is enough to consider the case where $\mu(\{\omega$ : $f(\omega) \neq 0\})>0$. Then, since $T$ is ergodic, we have

$$
\bigcup_{n=0}^{\infty} T^{-n}\{\omega: f(\omega) \neq 0\}=\Omega(\bmod \mu)
$$

and thus by Property (B) there exists a set $A_{1} \in \mathcal{A}$ with $A_{1} \subset A$ and $\mu\left(A_{1}\right)>0$ for which $h \chi_{A_{1}} \in L$. This shows that we may assume from the start that $h \chi_{A} \in L$.

Now, since $\left(h \circ T^{j}\right) \chi_{A}=\left(S_{j} f\right) \chi_{A}+h \chi_{A}$ for $j \geq 1$,

$$
\begin{aligned}
\frac{1}{n} \sum_{j=1}^{n}\left\|\left(S_{j} f\right) \chi_{A}\right\|_{L}+\left\|h \chi_{A}\right\|_{L} & \geq \frac{1}{n} \sum_{j=1}^{n}\left\|\left(h \circ T^{j}\right) \chi_{A}\right\|_{L} \\
& =\frac{1}{n} \sum_{j=1}^{n}\left\|h\left(\chi_{A} \circ T^{-j}\right)\right\|_{L} \quad \text { (by Property (D)) } \\
& \geq\left\|h\left(\frac{1}{n} \sum_{j=1}^{n} \chi_{A} \circ T^{-j}\right)\right\|_{L}
\end{aligned}
$$

If we let, for $n \geq 1$,

$$
d_{n}(\omega)=\inf _{m \geq n} \frac{1}{m} \sum_{j=1}^{m} \chi_{A}\left(T^{-j} \omega\right)
$$

then, by the Birkhoff ergodic theorem together with the ergodicity of $T$,

$$
0 \leq d_{1}(\omega) \leq d_{2}(\omega) \leq \ldots \rightarrow \mu(A)>0 \quad \text { for } \mu \text {-a.e. } \omega \in \Omega .
$$

Hence, $\mu(A)|h|(\omega)=\lim _{n \rightarrow \infty} d_{n}(\omega)|h|(\omega)$ for $\mu$-a.e. $\omega \in \Omega$. Furthermore,

$$
\left\|d_{n} h\right\|_{L} \leq\left\|h\left(\frac{1}{n} \sum_{j=1}^{n} \chi_{A} \circ T^{-j}\right)\right\|_{L} \leq \frac{1}{n} \sum_{j=1}^{n}\left\|\left(S_{j} f\right) \chi_{A}\right\|_{L}+\left\|h \chi_{A}\right\|_{L} .
$$

Thus, by (31), there exists a subsequence $\left(n^{\prime}\right)$ of $(n)$ such that $\left\|d_{n^{\prime}} h\right\|_{L} \leq K+$ $\left\|h \chi_{A}\right\|_{L}+1$ for all $n^{\prime}$. By Properties (C) and (B) it follows that $\mu(A) h \in L$, and hence the proof is complete.

REMARK 2. If the ergodicity of $T$ is not assumed in Theorem 2, then inequality (31) with $A=\Omega$ implies the same conclusion of the theorem.

To see this, we first notice that the above proof, together with Remark 1 , shows that there exists a real-valued measurable function $h$ on $\Omega$ such that $f(\omega)=h(T \omega)-h(\omega)$ for $\mu$-a.e. $\omega \in \Omega$. Since $\left|S_{j} f\right|=\left|h \circ T^{j}-h\right| \geq\left|h \circ T^{j}\right|-|h|$ 
for $j \geq 1$, it follows that

$$
\frac{1}{n} \sum_{j=1}^{n}|h| \circ T^{j} \leq \frac{1}{n} \sum_{j=1}^{n}\left|S_{j} f\right|+|h| .
$$

Therefore, the Birkhoff ergodic theorem implies that

$$
\lim _{n \rightarrow \infty} \frac{1}{n} \sum_{j=1}^{n}|h|\left(T^{j} \omega\right) \leq \liminf _{n \rightarrow \infty} \frac{1}{n} \sum_{j=1}^{n}\left|S_{j} f(\omega)\right|+|h(\omega)|<\infty
$$

for $\mu$-a.e. $\omega \in \Omega$, and hence the limit

$$
h_{\infty}(\omega)=\lim _{n \rightarrow \infty} \frac{1}{n} \sum_{j=1}^{n} h\left(T^{j} \omega\right)
$$

exists and is finite for $\mu$-a.e. $\omega \in \Omega$. Then

$$
\lim _{n \rightarrow \infty} \frac{1}{n} \sum_{j=1}^{n} S_{j} f(\omega)=h_{\infty}(\omega)-h(\omega)
$$

for $\mu$-a.e. $\omega \in \Omega$. Since $h_{\infty}(\omega)=h_{\infty}(T \omega)$ for $\mu$-a.e. $\omega \in \Omega$, it follows that the function $h_{0}(\omega):=h(\omega)-h_{\infty}(\omega)$ satisfies $f(\omega)=h_{0}(T \omega)-h_{0}(\omega)$ for $\mu$-a.e. $\omega \in \Omega$. Furthermore,

$$
\left|h_{0}(\omega)\right| \leq \liminf _{n \rightarrow \infty} \frac{1}{n} \sum_{j=1}^{n}\left|S_{j} f(\omega)\right|<\infty
$$

for $\mu$-a.e. $\omega \in \Omega$. Since inequality (31) with $A=\Omega$ shows that the function $f_{\infty}(\omega)=\liminf _{n \rightarrow \infty}(1 / n) \sum_{j=1}^{n}\left|S_{j} f(\omega)\right|$ belongs to $L$ (see $(35)$ ), we get $h_{0} \in L$ by Property (B). This establishes the desired conclusion.

\section{References}

[1] A. I. Alonso, J. Hong and R. Obaya, Absolutely continuous dynamics and real coboundary cocycles in $L^{p}$-spaces, $0<p<\infty$, Studia Math. 138 (2000), 121-134.

[2] I. Assani, Note on the equation $Y=(I-T) X$ in $L^{1}$, Illinois J. Math. 43 (1999), 540-541.

[3] I. P. Cornfeld, S. V. Fomin and Ya. G. Sinai, Ergodic Theory, Springer, Berlin, 1982.

[4] P. R. Halmos, Measure Theory, Van Nostrand, New York, 1950.

[5] H. Helson, Note on additive cocycles, J. London Math. Soc. (2) 31 (1985), 473-477.

[6] - The Spectrum Theorem, Lecture Notes in Math. 1227, Springer, Berlin, 1986.

[7] E. Hewitt and K. A. Ross, Abstract Harmonic Analysis. Vol. II, Springer, Berlin, 1970.

[8] R. A. Hunt, On L $(p, q)$ spaces, Enseign. Math. 12 (1966), 249-276.

[9] M. A. Krasnosel'skiı̌ and Ya. B. Rutickiŭ, Convex Functions and Orlicz Spaces, Noordhoff, Groningen, 1961.

[10] U. Krengel, Ergodic Theorems, de Gruyter, Berlin, 1985. 
[11] M. Lin and R. Sine, Ergodic theory and the functional equation $(I-T) x=y$, J. Operator Theory 10 (1983), 153-166.

[12] H. L. Royden, Real Analysis, 3rd ed., Macmillan, New York, 1988.

[13] R. Sato, A remark on real coboundary cocycles in $L^{\infty}$-space, Proc. Amer. Math. Soc., to appear.

Department of Mathematics

Okayama University

Okayama, 700-8530 Japan

E-mail: satoryot@math.okayama-u.ac.jp

Received April 23, 2002

Revised version October 21, 2002 